\title{
CONFLICTOS POR EL USO DEL AGUA EN UNA REGIÓN ÁRIDA: CASO TACNA, PERÚ
}

\author{
CONFLICTS OVER THE USE OF WATER IN AN ARID REGION: \\ CASE OF TACNA, PERU
}

\author{
Edwin Pino V.
}

\begin{abstract}
En la región Tacna al sur de Perú y norte de Chile, emplazada en la cabecera del Desierto de Atacama, la disponibilidad de agua para cubrir las demandas para uso poblacional y agrícola son cada vez más escasas. Basados en una revisión sistemática documentaria acerca de sucesos en la zona de estudio, establecemos que no solo nos enfrentamos a problemas de aridez y cambio climático, en los últimos años se han presentado problemas sociales, conflictos de uso y se transgrede la ley de Recursos Hídricos, sin darse prioridad de uso de acuerdo con lo que ella estipula. En este trabajo se busca caracterizar los conflictos por el uso del agua en esta región, emplazada en la cabecera del Desierto de Atacama, en función de los actores que intervienen. En la zona altoandina el conflicto se da entre entes gubernamentales encargados del trasvase de agua hacia la costa y los comuneros; además de conflictos internacionales. El río Maure conforma el sistema Titicaca, Desaguadero, Lago Poopó y Salar de Coipasa (TDPS) que incluye a Perú y Bolivia y se administra bajo la Autoridad Binacional Autónoma del Sistema Hídrico del Lago Titicaca, Río Desaguadero, Lago Poopó, Salar de Coipasa, denominada como ALT. El agua subterránea, con problemas de desbalance, en la zona costera permite cubrir las demandas poblacionales y el riego, se dieron dispositivos legales respecto de vedas, y posteriormente se emite un decreto supremo, contradictorio a las vedas para formalización de usuarios, esto ha generado mayor conflicto entre los administradores del agua, usuarios formalizados y los que pretendían formalizarse.
\end{abstract}

Palabras claves: Conflictos, uso del agua, zonas áridas, Desierto Atacama.

In the Tacna region in southern Peru and northern Chile, located at the head of the Atacama Desert, the availability of water to meet the demands for population and agricultural use is increasingly scarce. Based on a systematic documentary review on events in the study area, we establish that we not only face problems of aridity and climate change, in recent years there have been social problems, conflicts of use, the law of Water Resources is violated, without giving priority of use according to what it stipulates. In this work, we seek to characterize the conflicts over the use of water in this region, located at the head of the Atacama Desert, according to the actors involved. In the high Andean zone, the conflict occurs between government entities in charge of the transfer of water to the coast and the community members; in addition to international conflicts. The Maure River forms the Titicaca, Desaguadero, Lake Poopó and Salar de Coipasa (TDPS) system that includes Peru and Bolivia and is administered under the Binational Autonomous Authority of the Water System of Lake Titicaca, Río Desaguadero, Lake Poopó, Salar de Coipasa, referred to as ALT. Underground water with imbalance problems, in the coastal zone allows meeting population demands and irrigation, legal provisions were issued on closures, and later a supreme decree was issued, contradictory to the closures for the formalization of users, this has generated greater conflict between water administrators, formalized users and those who wanted to formalize.

Key words: Conflicts, water use, arid zones, Atacama Desert.

\section{Introducción}

Aproximadamente el 97,5\% del agua en la tierra es agua salada o agua que se ha contaminado, del 2,5\% restante, casi el 70\% está congelado en glaciares y casquetes polares. Menos del 0,01\% del agua en el mundo está disponible para uso humano en lagos, ríos, embalses y acuíferos de fácil acceso (Levy and Sidel, 2011). La edición 2018 del Informe de las Naciones Unidas acerca del desarrollo de los recursos hídricos en el mundo declaró que casi 6 mil millones de personas sufrirán de escasez de agua potable para el 2050 y la escasez de agua dulce se percibe cada vez más como un riesgo sistémico mundial (Boretti and Rosa, 2019; Kopnina and Washington, 2016; Mekonnen and Hoekstra, 2016). El crecimiento poblacional a nivel mundial seguirá afectando al medio ambiente generando grandes impactos en los ecosistemas, especialmente en las zonas costeras (Malanson and Alftine, 2016). Estas regiones costeras, especialmente las que se encuentran en regiones áridas, tienen un clima y un entorno ecológico frágil y sensible (Wang and Qin, 2017; Wardropper and Rissman, 2019; West et al., 2017;

* Universidad Nacional Jorge Basadre Grohmann. Tacna, Perú. Correo electrónico: epinov@unjbg.edu.pe ORCID: https:// orcid.org/0000-0001-7432-4364 
Zhang, 2000). La región de estudio se encuentra ubicada en la cabecera del Desierto de Atacama que es uno de los principales desiertos hiperáridos del mundo (Stein et al., 2013); en consecuencia, esta región tiene clima hiperárido, y se encuentra en el extremo sur de Perú y norte de Chile y su hiperaridez se atribuye a su ubicación subtropical (Pino et al., 2017, 2019b; Pino V. et al., 2018, Garreaud et al., 2003; Garreaud et al., 2010). El paisaje de Atacama se caracteriza por ser un espacio relacional en el que confluyen múltiples formas de apropiación social de la naturaleza, que establecen relaciones de cohabitación o conflicto (Manríquez et al., 2019). Las relaciones geográficas en esta región requieren un análisis espacial integrado de la región sudamericana, que supere las ficticias divisiones nacionales de los climas, sustituyéndola por análisis que contribuyan a su predicción y mejoramiento de la calidad de vida de los habitantes enfrentados a las variabilidades y cambios climáticos (Mendonça, 2017). En el norte de Chile el factor climático ha actuado constantemente, meteorizando y erosionando los materiales, dando como resultado formas derivadas de procesos gravitacionales, fluviales y, en menor medida, periglaciales (Rodríguez et al., 2017). La creciente escasez de agua provoca una variedad de presiones en la producción agrícola, debido a la actual y creciente demanda de alimentos (Booker and Trees, 2020).

Se pueden establecer tres ejes en los conflictos por el agua: (1) el abasto urbano y las dinámicas rural-urbanas de usos de agua múltiples; (2) la organización de usuarios y problemáticas socioambientales asociadas con la agricultura de riego, y (3) la hidropolítica internacional (García, 2016). En Perú, la construcción de una nueva arquitectura institucional del agua ha influido en los actores y las dinámicas sociopolíticas relacionados con la gestión de los recursos hídricos, en medio de estos procesos de institucionalización y conflicto aparecen nuevos actores y otros que estaban relegados toman mayor protagonismo, produciendo un escenario complejo para la gestión (Oré-Vélezy and GengMontoya, 2015). El problema del uso del agua, en todos sus términos, altera y excede las leyes acerca de los regímenes de gestión y las limitaciones en la disponibilidad de los recursos hídricos, si no se toman acciones inmediatas por parte del aparato gubernamental, la crisis del sistema podría volverse irreversible con los consecuentes daños económicos que esto conlleva (Pino V. et al., 2018).
En este trabajo se busca caracterizar los conflictos por el uso del agua en esta región, emplazada en la cabecera del Desierto de Atacama, en función de los actores que intervienen. El ámbito de estudio específicamente corresponde a la región de Tacna y se busca interrelacionar los conflictos sociales con las acciones gubernamentales que se establecen para suplir el déficit de agua.

\section{Materiales y métodos}

\section{Descripción del área de estudio}

La región se encuentra ubicada al sur de Perú y norte de Chile (Figura 1), pertenece a la cabecera del Desierto de Atacama, caracterizado como el más árido del mundo (Houston and Hartley, 2003; Ritter et al., 2019). En esta región, los restos conservados de actividad fluvial constituyen una prueba de las condiciones cambiantes de los límites, el Desierto de Atacama en el norte de Chile es un testigo mundial de aridez, por lo que la historia de las redes de arroyos relictos en esta región es un registro de cómo se desarrollan los paisajes en condiciones extremas (Binnie et al., 2020). La zona andina corresponde al sector centro-oeste del Altiplano, en el que la precipitación acumulada anual es relativamente baja (300-700 mm/año) y la variabilidad temporal es muy marcada, períodos húmedos de diciembre a marzo y muy secos de abril a noviembre (Garreaud et al., 2003; Garreaud et al., 2010; Ritter et al., 2019). El desierto costero no muestra tendencias significativas, lo que implica la mantención de las condiciones hiperáridas (Sarricolea et al., 2017). La región costera presenta un clima desértico; por esta razón, las precipitaciones anuales son escasas y hasta nulas (Pino et al., 2020, 2019a, 2019b, 2017; Pino V., 2019; Pino V. et al., 2018). El clima en la región costera de Tacna es templado, desértico, con amplitud térmica moderada. La media anual de temperatura máxima y mínima (periodo $1950-2019$ ) es $23,6^{\circ} \mathrm{C}$ y $12,7^{\circ} \mathrm{C}$, respectivamente. Estas características climáticas hacen que la región presente déficit hídrico, no se cubren las demandas de agua para los diferentes usos, lo que viene generando conflictos sociales por su uso a nivel regional, nacional y mundial.

\section{Conflictos por el agua en el mundo}

Según Hatami and Gleick (1994), la disputa fronteriza Lagash-Umma es la más antigua que 


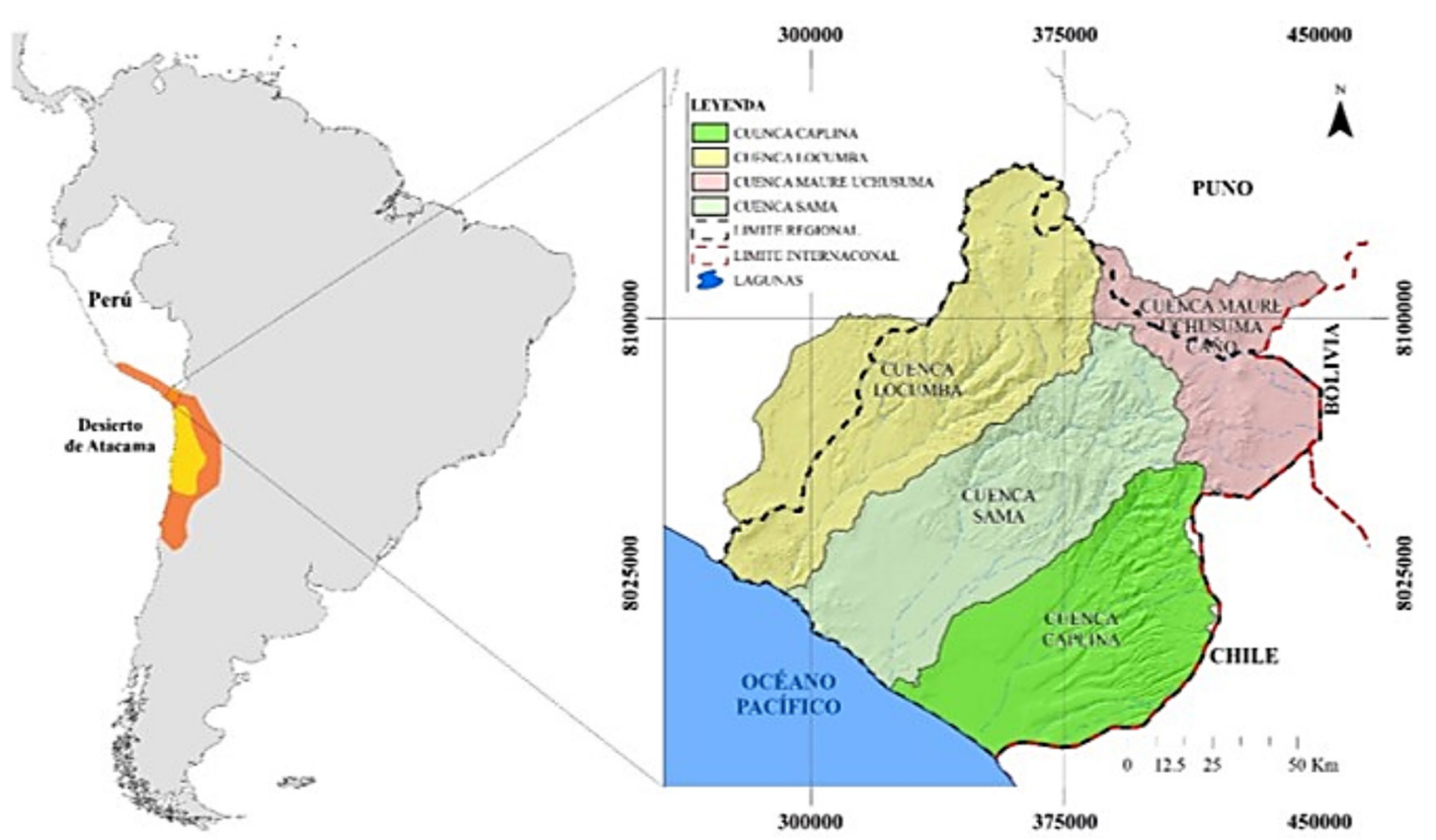

Figura 1. Mapa de ubicación de la zona de estudio.

se ha podido documentar, data de 2.500 a.C., se utilizó armas, se dio en Mesopotamia, Asia occidental; comienza la disputa por la región de Gu'edena. Urlama, rey de Lagash desvía el agua de esta región a los canales limítrofes, secando las zanjas limítrofes para privar a Umma de agua. Los conflictos por el agua, dentro de los países y entre países, están aumentando drásticamente; en el mundo se registraron conflictos de alcance internacional con violencia, un total de 22 entre 1900 y 1959; 38 entre 1960 y 1989 y 83 entre 1990 y 2007 (Levy and Sidel, 2011). Estas cifras son indicadores de un creciente escenario de conflictos con promedios que van desde 0,37 hasta 4,61 conflictos por año.

El estrés y la escasez de agua han afectado y seguirán afectando la estabilidad de las comunidades. Una descripción general de los desafíos de la seguridad hídrica mundial indica profundas dificultades y posibles puntos conflictivos (Kreamer, 2012). El agua ha sido, viene siendo y será a futuro una herramienta estratégica, objetivo étnico y religioso; es fundamental para la vida humana y las especies que habitan en el planeta tierra; $y$ a su vez es fundamental para las actividades económicas. En este sentido, desde tiempos pasados, los conflictos por el agua han tenido lugar entre las sociedades humanas, se espera que ha futuro los grandes conflictos bélicos sean por el agua. Los conflictos pasan de ser locales a la escala regional, nacional e internacional, basados en un concepto conocido como importación y exportación de agua. En los sistemas hídricos no se diferencian fronteras geopolíticas y en muchos casos resultan ser de alcance multinacional, siendo este el contexto de muchos casos en Sudamérica, como por ejemplo entre Argentina, Brasil y Paraguay, Perú y Bolivia, Perú, Ecuador y Colombia, entre otros. Los conflictos de distribución entre países vecinos situados a orillas de ríos transfronterizos, muestran claramente cómo el acceso común al agua puede convertirse en una cuestión de derechos territoriales y poder político (Zeitoun et al., 2020).

Los conflictos por el agua en Perú y en Sudamérica se han incrementado considerablemente en los últimos años, alcanzando altos niveles de complejidad e impacto, vienen afectando las economías, los ámbitos políticos, la estabilidad social, las poblaciones y el ambiente.

\section{La escasez de agua como factor de conflictos}

La historia del mundo está llena de tensiones provocadas por la escasez de recursos naturales: oro, diamantes y petróleo. Sin embargo, el agua, 
que hasta ahora no había sido considerada un factor de conflicto, con el cambio climático se convierte en "el oro azul" de este siglo (Nieto, 2011). En la actualidad, la situación ambiental y ecológica mundial, sin duda uno de los problemas de mayor trascendencia es el referente al agua, atribuido a la variabilidad en su distribución geográfica, tanto en el tiempo como en el espacio (Peña, 2007); adicionalmente a esta situación, las decisiones políticas y económicas determinan nuestra relación social respecto del agua. En muchas regiones del mundo el Estado no asume su papel, no se cumplen las disposiciones legales vigentes y los usuarios legalizados y no legalizados generan enfrentamientos con resultados catastróficos. La probabilidad que se produzcan conflictos por el agua podría aumentar a medida que las poblaciones continúen creciendo y el cambio climático continúe manifestándose (Petersen-Perlman et al., 2017). Una regla general se da cuando un recurso se hace escaso, esto se convierte en un detonante de conflictos, siendo el agua un elemento que se acomoda perfectamente a esta regla. La región de estudio se caracteriza por su hiperaridez, las fuentes superficiales son de descargas muy bajas respecto de las cuencas de la zona centro y norte del país; la región norte presenta características marcadas con tendencia hacia un clima tropical, temperaturas muy elevadas sin marcadas diferencias en las estaciones del año, donde los volúmenes de agua en los ríos son normalmente altos. Según Pino et al. (2019b), la región de Tacna es la zona más árida de la costa peruana, caracterizada por la escasez de recursos hídricos, según la Figura 2, los ríos de la costa en esta región (Caplina, Sama y Locumba, de sur a norte) son los que tienen los caudales medios mensuales más bajos de toda la costa del país.

Es una preocupación latente y permanente en el tiempo, que las cuencas de la región hidrográfica Pacífico del Perú, especialmente la región sur, presenten los mayores déficits y vean cuestionadas sus posibilidades de desarrollo económico por la falta de agua. Esta situación limita enormemente las posibilidades de desarrollo, el país es agrícola y minero, como actividades económicas principales, lo que se ve amenazado y afectado por la falta de agua y por los conflictos sociales que acarrea la implementación de proyectos hidráulicos de trasvase que se conceptualizan y formulan como medida para atenuar el déficit hídrico en la región costera sur del país. Según Baum et al. (2016), se espera que la explotación de fuentes alternativas de agua aumente en las próximas décadas en países con estrés hídrico con un rápido crecimiento de la población, especialmente en regiones donde se espera una mayor disminución de la disponibilidad natural de agua dulce debido al cambio climático.

\section{El uso del agua en el norte de Chile}

Es imperativo establecer la tendencia respecto del uso del agua; el uso consuetudinario del agua por los pueblos indígenas del desierto de Atacama ha sido reconocido como un ejercicio de derecho propio de pueblos indígenas en Chile, tanto a nivel de la doctrina como la jurisprudencia nacional e internacional (Díaz, 2020). Asimismo, en el norte de Chile, a pesar que el Estado exige que las aguas nadie puede tomarlas sin su autorización o concesión; estas siempre han sido utilizadas sin previa concesión (Vergara, 1998), esta situación se traduce en un sistema de uso de agua por usos y costumbres también conocido como uso consuetudinario del agua, como ocurre en muchos lugares del mundo y que normalmente se configura bajo un sistema establecido legalmente en los países y que se encuentra estipulado en su legislación.

Según Costa (2016), la regulación de las aguas en Chile es un tema candente para el derecho y las políticas públicas; empujado por varios conflictos sociales en torno a este bien, se hace inminente la necesidad de efectuar algunas modificaciones en esta área. Se identificaron las tres principales características del derecho de aguas enraizado en el Código de Aguas de 1981: la existencia de un mercado de derechos de aguas, la preponderancia de la gestión por parte de los usuarios y una escasa injerencia de la Administración del Estado.

\section{Sistemas transfronterizos en Sudamérica}

Según la UNESCO, en Sudamérica se han identificado 29 sistemas transfronterizos, entre los que se encuentran los sistemas Bolivia-Perú (14S-Titicaca) y Chile-Perú (18S Concordia/ Escritos-Caplina). El primero ubicado a más de $4.000 \mathrm{msm}$ es parte de un ecosistema sensible. Es utilizado para abastecimiento de agua potable, ganadería y agricultura. Su área es de $120.000 \mathrm{~km}^{2}$. Presenta clima frío y seco y población dispersa de aproximadamente un millón de habitantes. El segundo, Chile-Perú, es una zona árida y seca. 

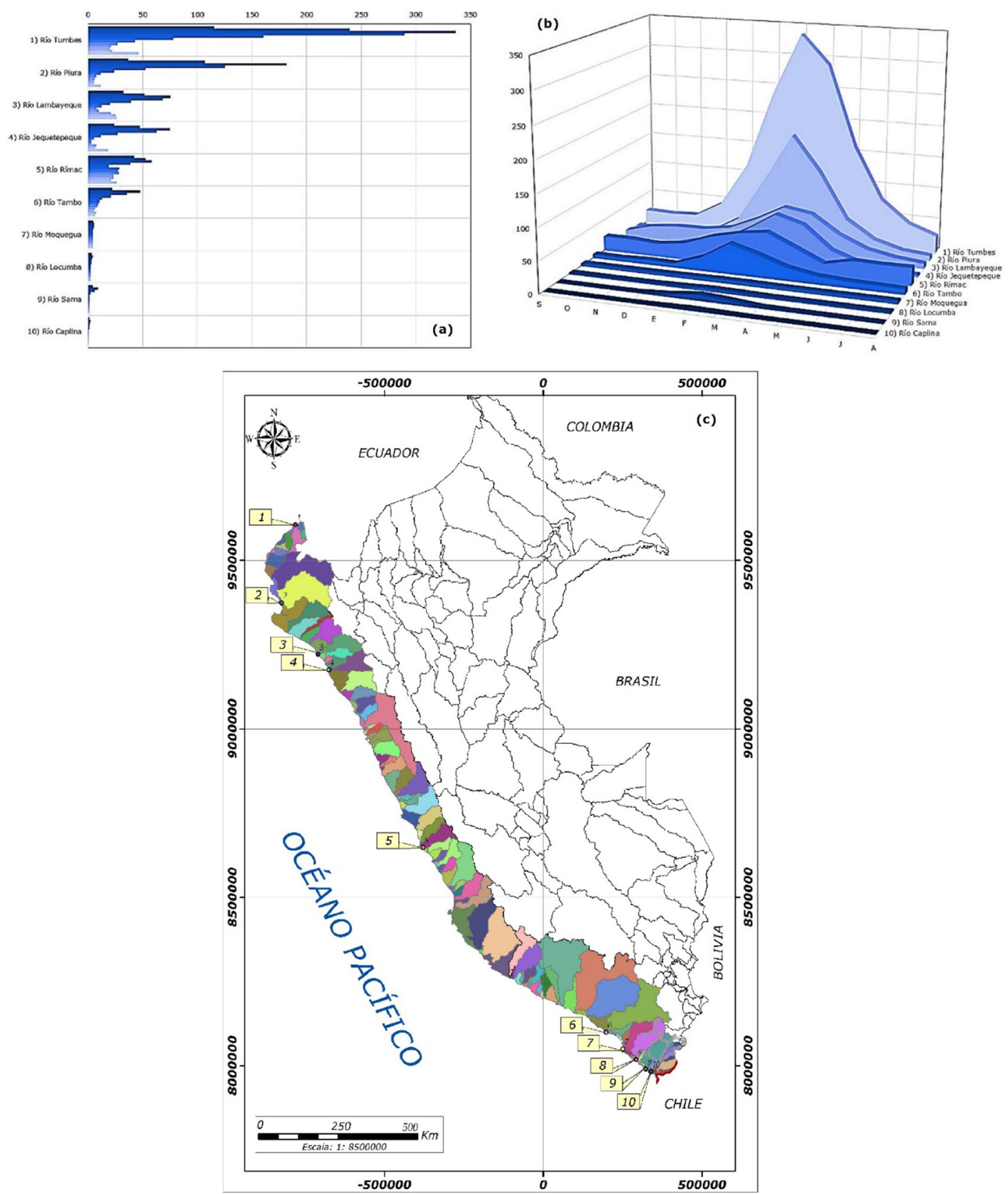

Figura 2. Descargas de los ríos y cuencas de la costa peruana.

Perú tiene estudios de detalle del acuífero. En el lado peruano la sobrexplotación está ocasionando la contaminación del acuífero (intrusión marina). Según Pino et al. (2020), en la actualidad el proceso de contaminación por intrusión marina se viene acrecentando debido a la sobreexplotación del agua subterránea, en este sentido, se plantean medidas correctoras para detener la degradación del sistema acuífero y lograr su recuperación.

\section{Tratados bilaterales en la región}

En esta región se cuenta con el tratado binacional Perú-Bolivia, basado en el lago Titicaca como 
elemento principal de un sistema hídrico endorreico en esta región, relacionado con los sistemas menores que corresponden al área de influencia de la cabecera del desierto de Atacama y que corresponden a la región hidrográfica del Pacífico. A este gran sistema se le conoce como el "sistema TDPS (con una superficie de $143.900 \mathrm{~km}^{2}$ )", siglas que aluden al lago Titicaca, al río Desaguadero, al lago Poopó y al salar de Coipasa. Su ámbito abarca una parte del sur de Perú, el occidente de Bolivia y una pequeña porción del norte de Chile interconectada con los ríos Maure y Desaguadero. La denominación de "sistema TDPS" viene desde la creación de la Autoridad Binacional Autónoma del Sistema Hídrico del Lago Titicaca, Río Desaguadero, Lago Poopó y Salar de Coipasa (más conocida por sus siglas ALT) entre Perú y Bolivia, en 1996, para el ordenamiento, manejo, control y protección en la gestión del agua en la zona. Resulta muy significativo que el ámbito de la ALT comprenda no solo el lago Titicaca y algunos de los ríos de curso sucesivo que lo integran, sino que abarque el sistema hidrográfico TDPS. Por ello, señalamos a la ALT como una de las experiencias más avanzadas en América del Sur. Según Maganda (2008), un caso que demuestra la eficacia de la cooperación, según el programa UNESCO, es la cooperación entre Bolivia y Perú por el manejo del lago Titicaca mediante la creación de una autoridad autónoma del agua.

\section{Conflictos sociales por el agua en la región}

Para identificar los conflictos sociales por el agua, se debe tener en cuenta los siguientes aspectos fundamentales: i) instrumentos internacionales para el reconocimiento del derecho al agua como derecho humano a nivel global; ii) la configuración constitucional y legal del agua en el país, así como la regulación vigente para el aprovechamiento del recurso, y iii) los principales instrumentos de planificación y organización del Estado respecto de la gestión del agua. Los conflictos sociales vinculados a los recursos hídricos tienen entre sus causas la afectación a la calidad, la cantidad y la oportunidad de la disponibilidad del agua. La intervención de los organismos estatales en la búsqueda de incrementar los recursos hídricos para la región costera del sur del país con la finalidad de cubrir las demandas insatisfechas poblacional, agrícola, minera, industrial, otras, viene generando conflictos sociales en la región de Tacna. La implementación de proyectos hidráulicos de trasvase de agua del altiplano a la región hidrográfica del Pacífico ha tenido oposición marcada por parte de los comuneros de la zona altoandina, esto se traduce en paralización de las obras de se vienen ejecutando tales como represamientos, bocatomas, canales, túneles, pozos de agua subterránea, entre otros.

\section{Resultados y Discusión}

Para combatir la desertificación, muchos gobiernos en el mundo han lanzado algunas Políticas y Proyectos de Control de la Desertificación durante las últimas décadas (Xu et al., 2017). En la región de Tacna, debido a la baja disponibilidad hídrica, desde hace más de 40 años se formularon dos proyectos hidráulicos de trasvase de agua de la cuenca altoandina del río Maure hacia las cuencas de la región hidrográfica del Pacífico. La cuenca Maure se ubica en la zona altiplánica y discurre sus aguas hacia la región hidrográfica del Titicaca, insertada en el gran sistema Titicaca, Desaguadero, lago Poopó y salar de Coipasa (TDPS) (Figura 3); el lago Titicaca constituye su elemento regulador que al nivel normal de su superficie de agua de $3.810 \mathrm{msm}$ ocupa una extensión de $8.400 \mathrm{~km}^{2}$ y embalsa un volumen de $932 \mathrm{~km}^{3}$; este sistema a pesar de ser muy extenso tiene una alta sensibilidad hídrica. La baja disponibilidad hídrica en la región conlleva a la generación de proyectos hidráulicos de trasvase de agua de la región altiplánica del Titicaca hacia la región hidrográfica del Pacífico, lo que ha provocado conflictos sociales.

\section{Conflictos por los proyectos hidráulicos de trasvase}

Los proyectos denominados Kovire y Vilavilani proyectan trasvasar agua de la región altoandina hacia la región del Pacífico, el primero hacia la cuenca Sama y luego Locumba para afianzar la laguna Aricota (ubicada en el departamento de Tacna, provincia de Candarave, distrito de Curibaya, Perú, a una altitud de $2800 \mathrm{msm}$, con un volumen total de almacenaje de $904 \mathrm{hm}^{3}$, siendo los ríos Salado y Callazas sus tributarios) y el segundo para dotar de agua para consumo poblacional y riego en la ciudad y valle de Tacna. En torno a estos proyectos se han generado varios conflictos con las comunidades campesinas de la zona altoandina, tanto de las regiones Tacna y Puno, además de algunos 

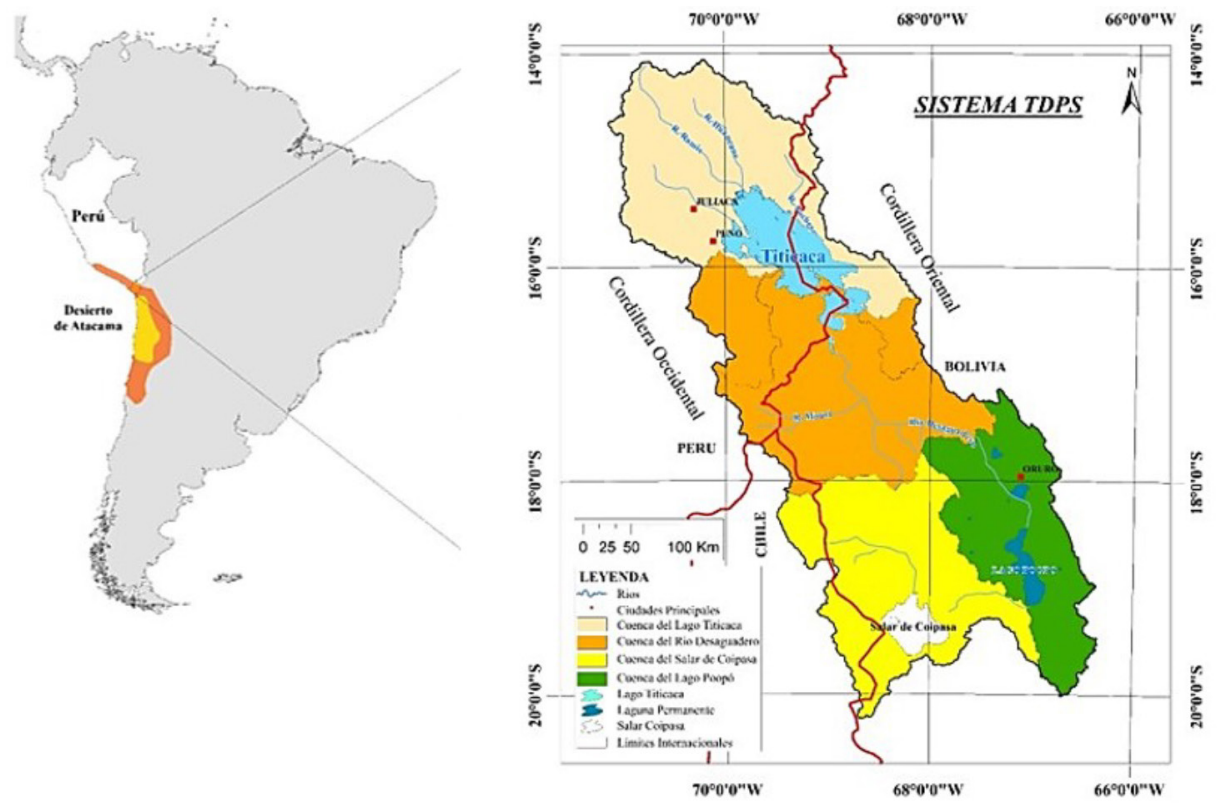

Figura 3. Sistema Titicaca, Desaguadero, lago Poopó y salar de Coipasa.

conflictos de orden internacional en el 2010, que llevó a reuniones entre cancillerías para socializar temas referidos al tratamiento internacional del agua del río Maure, que es la fuente principal de estos proyectos, sigue su curso internacional hacia su confluencia con el río Desaguadero en territorio boliviano en la localidad de Calacoto, donde existe una estación de aforo para medición de caudales.

\section{Proyecto Kovire}

Corresponde a un proyecto de trasvase de agua conceptualizado solo para épocas de lluvia con fines de afianzamiento de la laguna Aricota, la misma que en 1996 tuvo un descenso hasta casi llegar al agotamiento. La estructura principal del proyecto la constituye un túnel llamado "Kovire", que entró en operación en 1994, con sección tipo baúl, $8,4 \mathrm{~km}$ de longitud a una altitud de $4.400 \mathrm{msm}$ y capacidad máxima de conducción de $13 \mathrm{~m}^{3} / \mathrm{s}$. Esta estructura permite derivar agua del río Ancoaque, tributario del Maure en las nacientes de este, hacia la cuenca Sama, para luego ser captada en el dique Cano y conducidas por el canal Cano-Salado hacia el túnel Ichicollo cuya longitud es menor a $1 \mathrm{~km}, \mathrm{y}$ finalmente descargar sus aguas hacia el río Salado y este en la laguna Aricota (Figura 4). Los conflictos en este proyecto se suscitaron por la intervención territorial acerca de cursos de agua naturales de los ecosistemas altoandinos, especialmente en la parte de territorio de la región Puno.

\section{Proyecto Vilavilani}

Este proyecto hidráulico es concebido en sus inicios para usos múltiples, igualmente que el anterior, conceptualiza trasvase de agua en épocas de lluvia, para uso poblacional, agrícola y producción de energía eléctrica en un sistema escalonado de centrales hidroeléctricas. Actualmente se encuentra construido en forma parcial, el canal de conducción abierto está concluido, así como la batería de pozos de agua subterránea El Ayro y estructuras conexas, queda pendiente la conexión del punto de captación bocatoma Vilachaullani al canal Calachaca (Figura 5).

En el proceso de ejecución de este proyecto se suscitaron ciertos conflictos entre los comuneros y las instituciones estatales, la construcción del canal Vilachaullani-Calachaca-Chuapalca ha sido declarado de necesidad, utilidad pública y de interés nacional, mediante el Decreto de Urgencia 018-2019, que establece medidas extraordinarias para la implementación de los proyectos priorizados en el Plan Nacional de Infraestructura para la Competitividad. Al margen de las prioridades de uso establecidas en la ley de Recursos Hídricos 


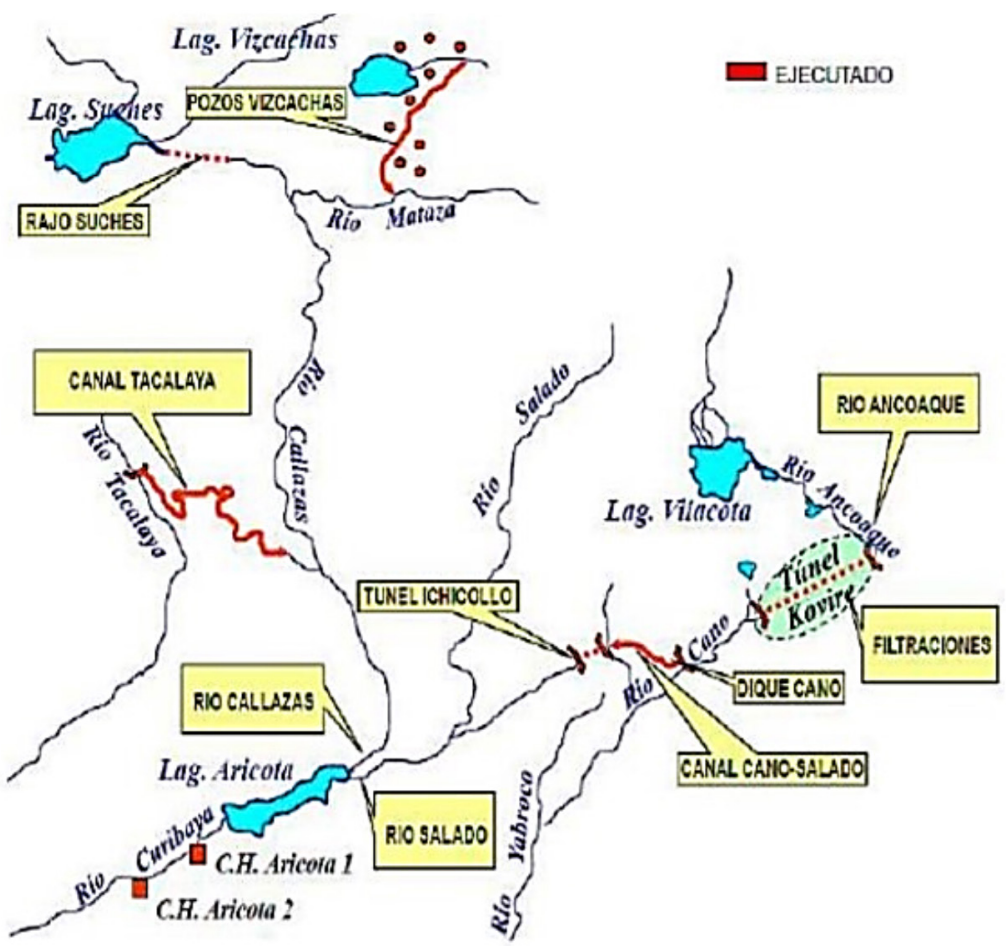

Figura 4. Esquema del Proyecto Kovire.

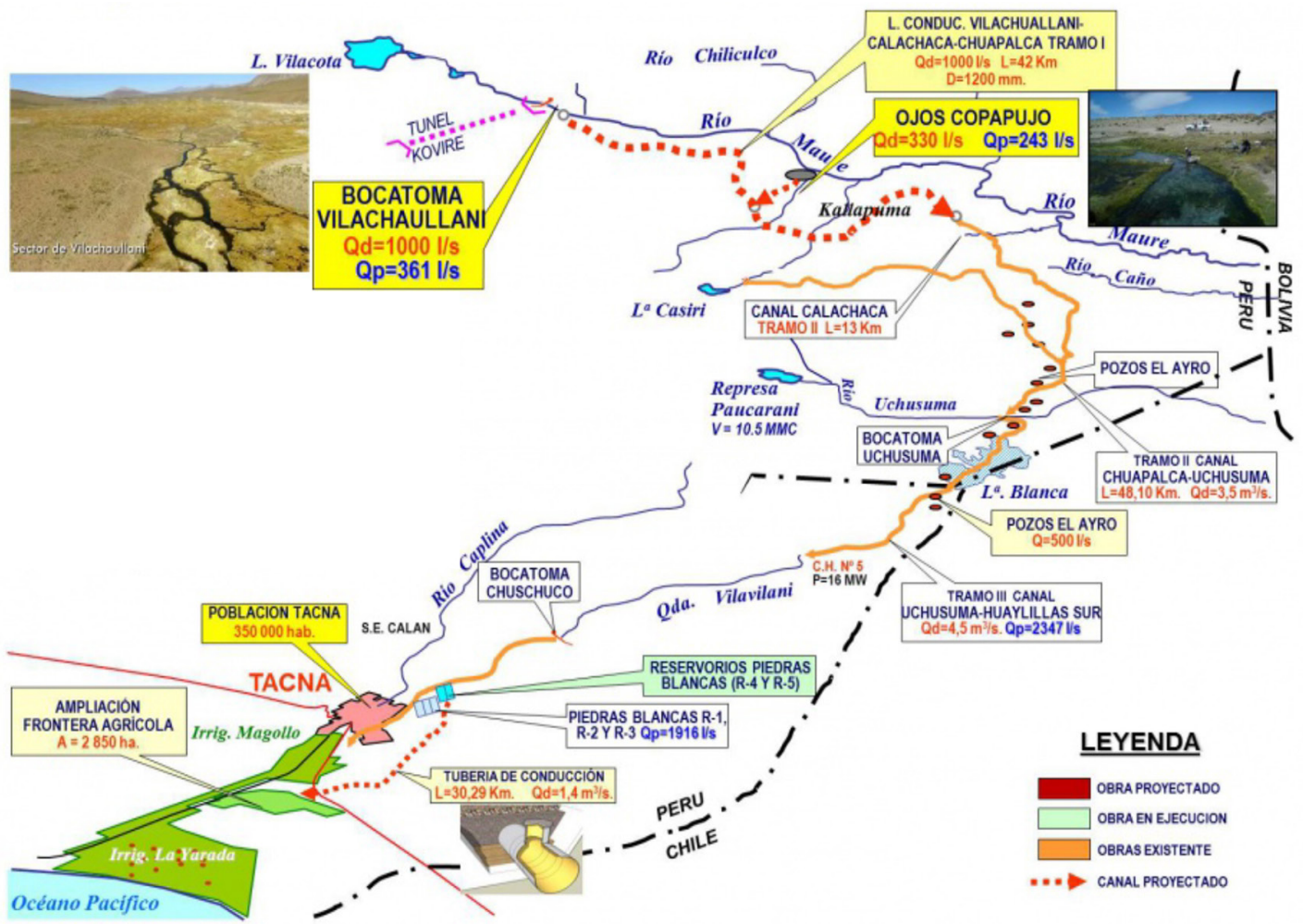

Figura 5. Esquema del Proyecto Vilavilani. 
$\mathrm{N}^{\mathrm{o}} 29338$, los conflictos son frecuentes, inclusive habiéndose propuesto que la captación y trasvase de agua es únicamente en épocas de lluvia.

\section{Conflictos por la explotación del agua subterránea}

Según Abotalib et al. (2016), los regímenes paleoclimáticos en el mundo alternaron entre períodos secos y húmedos a lo largo de la época del Pleistoceno, y es durante los períodos húmedos cuando se recargaron los acuíferos fósiles. Esta característica de acuífero fósil permite continuar explotando en forma desequilibrada estos acuíferos por muchos años, a pesar que la explotación en ellos supera ampliamente a la recarga. En esta región, los acuíferos corresponden a este tipo, el acuífero más grande de la región es conocido como La Yarada en la zona costera, parte baja de la cuenca Caplina, un acuífero sobreexplotado y con problemas de contaminación y deterioro de la calidad del agua por procesos de intrusión marina (Pino et al., 2019a, 2019b, 2017; Pino V., 2019; Pino V. et al., 2018). La crisis de gobernabilidad en el uso de aguas subterráneas en el acuífero costero La Yarada es un elemento que contribuye al agotamiento del agua subterránea (Pino V. et al., 2018).

Para este acuífero se han generado por varios años dispositivos legales gubernamentales acerca de vedas de perforación de nuevos pozos, como la R.M. $\mathrm{N}^{\circ}$ 696-98-AG que es ratificada el 2006, mediante D.S. $\mathrm{N}^{\circ}$ 065-2006-AG y contradictoriamente en el 2015 la política de gobierno genera una grave colisión emitiendo el D.S. N $^{\circ}$ 007-2015-MINAGRI, que autoriza la regularización de licencias de uso de agua. Esta situación representa un antecedente negativo de la política gubernamental en la administración y manejo del agua, se han producido enfrentamientos entre grupos de usuarios con licencia y los que no disponen de esta y también con los funcionarios de la autoridad local del agua (ALA). Se evidenció que, según la ley de recursos hídricos, no es posible otorgar una licencia de uso de agua a un sistema que está en déficit hídrico, por tanto, la dación del decreto supremo D.S. $\mathrm{N}^{\circ}$ 007-2015-MINAGRI no es aplicable en virtud a lo que dispone la misma ley.

En la región altoandina se han identificado acuíferos multicapa, en sistemas bastante complejos en los que se tiene agua de buena y mala calidad por la acción del geotermalismo de la zona. Los acuíferos identificados son: El Ayro hacia la frontera con Chile,
Kallapuma y Vizcachas; en estos tres acuíferos se perforaron pozos exploratorios que reportaron altas temperaturas en el agua, que llegan hasta $\operatorname{los} 85^{\circ} \mathrm{C}$ y contenidos de boro, arsénico y otros elementos fuera de los límites permisibles para consumo humano. En este caso, la situación de conflicto por su explotación se genera por los pozos que extraen agua de buena calidad, lo que involucra a comunidades campesinas de la zona y las que se encuentran hacia la margen izquierda del río Maure que corresponde a la región de Puno. Estas comunidades generan férrea oposición a la ejecución de los proyectos hidráulicos de trasvase de agua hacia la región costera de Tacna, con el objetivo de cubrir el consumo doméstico, el que tiene prioridad de uso según la ley de Recursos Hídricos peruana.

\section{Conclusiones}

Según la Ley de Recursos Hídricos de Perú (Ley $\mathrm{N}^{\circ}$ 29338), se configura el principio de prioridad al acceso al agua para la satisfacción de las necesidades primarias de la persona humana siendo prioritario por ser un derecho fundamental sobre cualquier uso, incluso en época de escasez. La región de Tacna se encuentra emplazada en una zona árida, ubicada en la cabecera del desierto de Atacama, tiene baja disponibilidad de agua, lo que ha generado por muchos años conflictos de uso en la zona altoandina, entre las entidades estatales y los comuneros de la zona, por los volúmenes programados de trasvase de agua para cubrir las demandas de uso poblacional y riego en la ciudad y valle de Tacna. A su vez se han registrado conflictos internacionales por el uso del agua del río Maure, conformante del sistema TDPS, entre Perú y Bolivia. Asimismo, surge una pugna permanente por el agua subterránea, que a su vez no tiene legislación específica en el país, en el caso del acuífero La Yarada, principal acuífero de la región, se dieron dispositivos legales sobre vedas, que fueron ratificadas y posteriormente el mismo ente gubernamental emite un decreto supremo totalmente contradictorio a la dación de las vedas con fines de formalización de usuarios, instrumento que no pudo ser aplicado a un sistema acuífero en déficit, ya que las licencias de uso de agua según la propia ley de Recursos Hídricos se otorgan cuando se demuestra superávit en el balance. Esta situación ha generado mayor conflicto entre los administradores del uso de agua, los usuarios formalizados y los que pretendían hacerlo. 


\section{Referencias Citadas}

Abotalib, A. Z., Sultan, M., and Elkadiri, R.

2016 "Groundwater processes in Saharan Africa: Implications for landscape evolution in arid environments". In EarthScience Reviews (Vol. 156, pp. 108-136). Elsevier B.V. https://doi.org/10.1016/j.earscirev.2016.03.004.

Baum, Z., Palatnik, R. R., Kan, I., and Rapaport-Rom, M. 2016 "Economic Impacts of Water Scarcity under Diverse Water Salinities". Water Economics and Policy, 2(1). https:// doi.org/10.1142/S2382624X15500137

Binnie, S. A.; Reicherter, K. R.; Victor, P.; González, G.; Binnie, A.; Niemann, K.; Stuart, F. M.; Lenting, C.; Heinze, S.; Freeman,

S. P. H. T. and Dunai, T. J.

2020 "The origins and implications of paleochannels in hyperarid, tectonically active regions: The northern Atacama Desert, Chile". Global and Planetary Change, 185. https:// doi.org/10.1016/j.gloplacha.2019.103083.

Booker, J. F., and Trees, W. S.

2020 "Implications of Water Scarcity for Water Productivity and Farm Labor". Water, 12(1), 308. https://doi.org/10.3390/ w12010308.

Boretti, A., and Rosa, L.

2019 "Reassessing the projections of the World Water Development Report". Npj Clean Water, 2(1), 1-6. https:// doi.org/10.1038/s41545-019-0039-9.

Díaz Campos, Karenn Alejandra.

2020 "Crisis del agua en el norte de Chile. derecho y cultura en los Andes. Sobre los efectos irracionales del derecho". Diálogo Andino (61), 67-79. https://dx.doi.org/10.4067/ S0719-26812020000100067.

García, R.

2016 "Las pugnas por el agua: políticas, gestores y actores sociales". In Con-temporánea (Issue 6). http://con-temporanea.inah.gob.mx/mirar_libros/rita_garcia_num6.

Garreaud, R. D., Molina, A., and Farias, M.

2010 "Andean uplift, ocean cooling and Atacama hyperaridity: A climate modeling perspective". Earth and Planetary Science Letters, 292(1-2), 39-50. https://doi.org/10.1016/j. eps1.2010.01.017.

Garreaud, R., Vuille, M., and Clement, A. C.

2003 "The climate of the Altiplano: Observed current conditions and mechanisms of past changes". Palaeogeography, Palaeoclimatology, Palaeoecology, 194(1-3), 5-22. https:// doi.org/10.1016/S0031-0182(03)00269-4.

Hatami, H. and Gleick, P.

1994 "Chronology of Conflict over Water in the Legends, Myths, and History of the Ancient Middle East". Environment, 3(36).

Houston, J., and Hartley, A. J.

2003 "The central andean west-slope rainshadow and its potential contribution to the origin of hyper-aridity in the Atacama Desert". International Journal of Climatology, 23(12), 1453-1464. https://doi.org/10.1002/joc.938.

Kopnina, H., and Washington, $\mathrm{H}$.

2016 "Discussing why population growth is still ignored or denied". Chinese Journal of Population Resources and Environment, 14(2), 133-143. https://doi.org/10.1080/10 042857.2016.1149296.

Kreamer, D. K.

2012 "The Past, Present, and Future of Water Conflict and International Security". Journal of Contemporary
Water Research \& Education, 149(1), 87-95. https://doi. org/10.1111/j.1936-704x.2012.03130.x.

Levy, B. S., and Sidel, V. W.

2011 "Water Rights and Water Fights: Preventing and Resolving Conflicts Before They Boil Over". American Journal of Public Health, 101(5), 778-780. https://doi.org/10.2105/ AJPH.2010.194670.

Maganda, Carmen

2008 “Agua dividida, agua compartida? Acuíferos transfronterizos en Sudamérica, una aproximación. Estudios Políticos". Antioquía, número 32, enero-junio 2008, pp. 171-194.

Malanson, G. P., and Alftine, K. J.

2016 "Ecological Impacts of Climate Change". In Biological and Environmental Hazards, Risks, and Disasters (pp. 397-426). Elsevier Inc. https://doi.org/10.1016/ B978-0-12-394847-2.00022-X.

Manríquez Tirado, Hermann; Mansilla Quiñones, Pablo y

Moreira Muñoz, Andrés

2019 "Hacia una conservación integrada del paisaje biogeocultural de Atacama". Diálogo Andino (60), 141-152. https://dx.doi.org/10.4067/S0719-26812019000300141

Mekonnen, M. M., and Hoekstra, A. Y.

2016 "Sustainability: Four billion people facing severe water scarcity". Science Advances, 2(2). https://doi.org/10.1126/ sciadv. 1500323

Mendonça, Magaly

2017 "Monzón Sudamericano: La integración de la circulación amazónica y altiplánica y las variabilidades climáticas del altiplano andino chileno". Diálogo Andino (54), 21-30. https://dx.doi.org/10.4067/S0719-26812017000300021.

Nieto, Nubia

2011 "La gestión del agua: tensiones globales y latinoamericanas". Política y Cultura (36), 157-176. Recuperado en 16 de marzo de 2021, de http://www.scielo.org.mx/scielo. php?script=sci_arttext\&pid=S0188-77422011000200007\&ln $\mathrm{g}=\mathrm{es} \&$ tlng=es.

Oré-Vélezy, María, Geng-Montoya, D.

2015 "Politicas públicas del agua en el Perú: viscisitudes para la creación del consejo de recursos hídricos de la cuenca IcaHuancavelica". Agricultura, Sociedad y Desarrollo, 12(3), 409-445. http://www.scielo.org.mx/scielo.php?script=sci_ar ttext\&pid=S1870-54722015000300007.

Peña García, Alejandra

2007 "Una perspectiva social de la problemática del agua". Investigaciones Geográficas (62), 125-137. Recuperado en 15 de marzo de 2021, de http://www.scielo.org.mx/scielo. php?script=sci_arttext\&pid=S0188-46112007000100008\&ln $\mathrm{g}=\mathrm{es} \&$ tlng=es.

Petersen-Perlman, J. D., Veilleux, J. C., and Wolf, A. T.

2017 "International water conflict and cooperation: challenges and opportunities". In Water International (Vol. 42, Issue 2, pp. 105-120). Routledge. https://doi.org/10.1080/025080 60.2017.1276041.

Pino V. E.; Ramos F. L.; Mejía M. J.; Chávarri V. E. y Ascensios T. D.

2020 "Medidas de mitigación para el acuífero costero La Yarada, un sistema sobreexplotado en zonas áridas". Idesia (Arica), 38(3), 21-31. https://doi.org/10.4067/ s0718-34292020000300021. 
Pino, E., Montalván, I., Vera, A., and Ramos, L.

2019a "Stomatal conductance and its relationship with leaf temperature and soil moisture in olive cultivation (Olea europaea L.), in the period of fruit ripening, in arid zones. La Yarada, Tacna, Perú”. Idesia, 37(4), 55-64. https://doi. org/10.4067/S0718-34292019000400055.

Pino, Edwin, Ramos, L.; Ávalos, O.; Tacora, P.; Chávarri, E.; Angulo, O.; Ascensios, D.; and Mejía, J.

2019b "Factors affecting depletion and pollution by marine intrusion in the La Yarada's coastal aquifer, Tacna, Peru". Tecnología y Ciencias del Agua, 10(5), 177-213. https:// doi.org/10.24850/j-tyca-2019-05-07.

Pino, Edwin, Tacora, P.; Steenken, A.; Alfaro, L.; Valle, A.;

Chávarri, E.; Ascencios, D.; y Mejía Marcacuzco, J. A.

2017 "Efecto de las características ambientales y geológicas sobre la calidad del agua en la cuenca del río Caplina, Tacna, Perú”. Tecnología y Ciencias del Agua, 8(6), 77-99. https://doi.org/10.24850/j-tyca-2017-06-06.

Pino V., E.

2019 "The La Yarada coastal aquifer, after 100 years of exploitation as a sustenance for agriculture in arid zones: A historical review". Idesia, 37(3), 39-45. https://doi. org/10.4067/S0718-34292019000300039.

Pino V., E.; Chávarri V. E. and Ramos F. L.

2018 "Governability and governance crisis its implications in the inadequate use of groundwater, case coastal aquifer of la yarada, Tacna, Perú". Idesia, 36(3), 77-86. https:// doi.org/10.4067/S0718-34292018005001301.

Ritter, B.; Wennrich, V.; Medialdea, A.; Brill, D.; King, G.; Schneiderwind, S.; Niemann, K.; Fernández-Galego, E.; Diederich,

J.; Rolf, C.; Bao, R.; Melles, M.; and Dunai, T. J.

2019 "Climatic fluctuations in the hyperarid core of the Atacama Desert during the past $215 \mathrm{ka}$ ". Scientific Reports, 9(1), 1-13. https://doi.org/10.1038/s41598-019-41743-8.

Rodríguez Valdivia, Alan; Albornoz Espinoza, Cristián y Tapia Tosetti, Alejandro

2017 "Geomorfología del área de Putre, andes del norte de Chile: acción volcánica y climática en su modelado". Diálogo Andino (54), 7-20. https://dx.doi.org/10.4067/ S0719-26812017000300007.

Sarricolea, Pablo; Meseguer Ruiz, Oliver, y Romero-Aravena, Hugo 2017 "Tendencias de la precipitación en el norte grande de Chile y su relación con las proyecciones de cambio climático". Diálogo Andino (54), 41-50. https://dx.doi. org/10.4067/S0719-26812017000300041.
Stein, B. A.; Staudt, A.; Cross, M. S.; Dubois, N. S.; Enquist, C.; Griffis, R.; Hansen, L. J.; Hellmann, J. J.; Lawler, J. J.; Nelson, E. J.; and Pairis, A.

2013 "Preparing for and managing change: Climate adaptation for biodiversity and ecosystems". In Frontiers in Ecology and the Environment (Vol. 11, Issue 9, pp. 502-510). John Wiley \& Sons, Ltd. https://doi.org/10.1890/120277.

Vergara Blanco, Alejandro

1998 "Bases y principios del derecho de aguas. En especial de los usos consuetudinarios". En: Revista Anuario, Facultad de Ciencias Jurídicas, Universidad de Antofagasta ( $\mathrm{s} / \mathrm{n}$ ) pp. 119-123.

Wang, Y. J., and Qin, D. H.

2017 "Influence of climate change and human activity on water resources in arid region of Northwest China: An overview". In Advances in Climate Change Research (Vol. 8, Issue 4, pp. 268-278). National Climate Center. https://doi.org/10.1016/j.accre.2017.08.004.

Wardropper, C. B., and Rissman, A. R.

2019 "Adaptations to extreme storm events by conservation organizations". Climatic Change, 152(1), 85-101. https:// doi.org/10.1007/s10584-018-2342-8.

West, J. M.; Courtney, C. A.; Hamilton, A. T.; Parker, B. A.; Julius, S. H.; Hoffman, J.; Koltes, K. H.; and MacGowan, P.

2017 "Climate-Smart Design for Ecosystem Management: A Test Application for Coral Reefs". Environmental Management, 59(1), 102-117. https://doi.org/10.1007/ s00267-016-0774-3.

Xu, D., Song, A., and Song, X.

2017 "Assessing the effect of desertification controlling projects and policies in northern Shaanxi Province, China by integrating remote sensing and farmer investigation data". Frontiers of Earth Science, 11(4), 689-701. https:// doi.org/10.1007/s11707-016-0601-4.

Zeitoun, M.; Mirumachi, N.; Warner, J.; Kirkegaard, M.; and

Cascão, A.

2020 "Analysis for water conflict transformation". Water International, 45(4), 365-384. https://doi.org/10.1080/02 508060.2019 .1607479 .

Zhang, B. G.

2000 "Asymptotic behavior of solutions of certain difference equations". Applied Mathematics Letters, 13(1), 13-18. https://doi.org/10.1016/S0893-9659(99)00138-X. 